SECTION VI

SPECTRA OF COSMIC X-RAY SOURCES

Chairman:

C. DE JAGER 


\title{
SPECTROSCOPIC TECHNIQUES IN X-RAY ASTRONOMY
}

\author{
LEON VAN SPEYBROECK \\ American Science and Engineering, Cambridge, Mass., U.S.A.
}

\section{Introduction}

The catalog of X-ray sources now includes types I and II super-novae remnants, at least one pulsar, other periodic or quasi-periodic sources, starlike objects which emit primarily in $\mathrm{X}$ rays, normal galaxies, radio galaxies, Seyfert galaxies, a quasar, and an apparently isotropic extragalactic background. There is ample evidence that X-ray emission is characteristic of many of the most interesting objects in astronomy, and the background may have cosmological implications. This should not be too surprising, since significant X-ray emission occurs whenever high energy electrons interact, and high energy electron production is usually associated with explosive phenomena.

The most useful energy range for X-ray observations extends from about $200 \mathrm{eV}$ to perhaps $10 \mathrm{keV}$. The low energy limit results from the absorption by the interstellar media, which of course varies from object to object; some typical cutoffs are given in Table I. The high energy limit, which is much more arbitrary, results from the usually observed rapidly decreasing emission with increasing energy, and also from the lack of

TABLE I

Low energy cut-off due to the interstellar medium

\begin{tabular}{ll}
\hline Extragalactic objects & E-cut-off \\
\hline Pole & $0.2 \mathrm{keV}$ \\
$60^{\circ}$ latitude & 0.3 \\
$30^{\circ}$ latitude & 0.44 \\
Galactic objects & \\
In the Plane & \\
$10 \mathrm{pc}$ & $0.12 \mathrm{keV}$ \\
$1 \mathrm{kpc}$ & 0.7 \\
$10 \mathrm{kpc}$ (toward galactic center) & $3 \mathrm{keV}$ \\
\hline
\end{tabular}

important characteristic emission or absorption features above this energy range. In many cases, however, observations outside of this energy range are required to definitively identify a dominant source mechanism.

The source mechanisms usually considered include synchrotron emission, inverse compton scattering, thermal emission from an optically thin hot gas, and black body radiation. Synchrotron emission and inverse compton scattering typically result in a power law spectrum extending over wide energy intervals. The thermal emission from a thin gas with normal cosmic abundance is dominated by line emission at lower temperatures and by thermal bremsstrahlung on hydrogen and helium nuclei at higher 


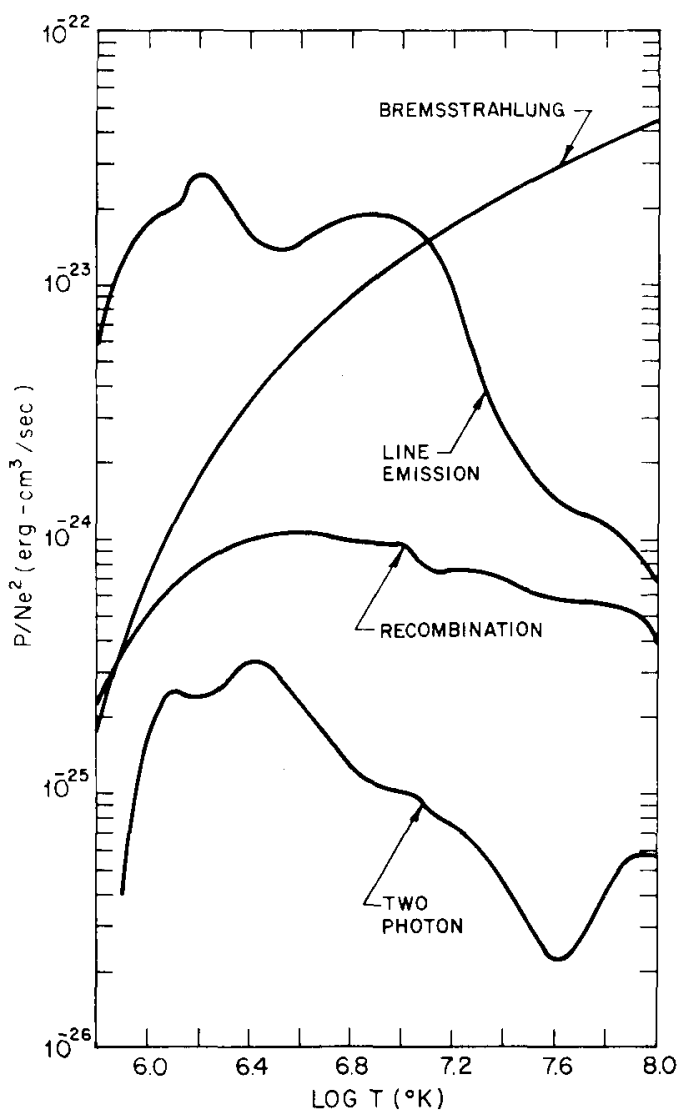

Fig. 1. The temperature dependance of the processes contributing to the emission of a hot thin plasma with coronal abundances; the values were taken from the calculation by Tucker and Koren (1971).

temperatures; the emission predicted by Tucker and Koren (1971) as a function of temperature, which is shown in Figure 1, illustrates this behavior. At lower temperatures, where line emission is important, we can expect to obtain the same information from stellar spectral data as from the excellent solar results presented at this conference. This will include measurements of elemental abundances, temperatures, densities, velocities, and turbulence or other large scale motions. The high resolution spectral observations of Sco XI by Pounds (1971) and by Kesterbaum et al. (1971) warn us, however, that at least this source has either unexpectedly weak or very broad emission lines. If the temperature is high enough so that thermal bremsstrahlung is dominant, then the spectrum will be of the form

$$
P(E) \approx g(E, T) \exp (-E / k T),
$$

where $g(E, T)$ is the effective Gaunt factor, and is of order unity. This form is such that the source temperature cannot be determined unless measurements extend to 
$E \approx k T$. Finally, if the source is optically thick, we obtain the limit of black body radiation, which has a more sharply peaked spectra than the emission from an optically thin gas.

The interstellar absorption significantly modifies the intrinsic source spectra; the X-ray absorption coefficients vary approximately as $E^{-8 / 3}$ between absorption edges, and so the optical depth can be approximated by $\tau(E) \approx\left(E_{\mathrm{a}}\right)^{-8 / 3}$. Low spectral resolution measurements are often expressed in terms of the effective cut-off energy $E_{\mathbf{a}}$. Higher resolution measurements also are sensitive to absorption edges and can be used to determine the abundances of the important elements in the interstellar media. Still higher resolution would enable measurements of the ionization state of the interstellar media.

Relatively crude measurements suffice to distinguish between the principal source models and to determine their few parameters if line emission is unimportant. The primary requirement is a sufficiently broad energy range of the measurements, at least of order $k T$ for the thermal sources. The resolution must also be small in terms of $k T$ and $E_{\mathrm{a}}$ in order to determine these parameters. These requirements are consistent with the properties of proportional counters, which have been the most commonly used detectors in X-ray astronomy because of their high efficiencies and because large sensitive areas are easily obtained. These qualities are necessary because of the low photon fluxes characteristic of $\mathrm{X}$-ray sources. We will discuss the proportional counter in detail because of its importance both in present $\mathrm{X}$-ray measurements and as the normal detector for the high resolution crystal instruments to be discussed below.

\section{Proportional Counters}

The essential parts of a proportional counter are a point or wire anode, counter gae, a cathode which may also bs the gas enclosure and structure, and an X-ray transmitting window. An X-ray photon is absorbed in the gas by the photo-electric process; the primary photo-electron loses its energy and creates electron-ion pairs in a small adjacent volume. These electrons drift towards the anode because of the electric field in the counter. In the large field region near the anode the electrons gain enough energy between collisions to ionize additional gas molecules, thus resulting in 'gas gain' or amplification. This amplification ranges from about $10^{3}$ to $10^{5}$ in most applications. The electrons traverse the multiplication region to the anode in about one nanosecond, and largely remain there as the electrostatic images of the lower mobility positive ions which remain in the gas. The positive ions then drift towards the cathode and allow the electrons to be collected by the external circuitry; for a localized initial event, the pulse shape is determined by the positive ion mobility. If a mixture of gasses is used, then charge exchange collisions will occur and the effective charge carrier will be the species with the lowest ionization potential.

The principal gas constituent is often a noble gas, since they are readily available with high atomic number and, therefore, large absorption coefficients, do not form negative ions, and result in a high gain for a given voltage. The high gain occurs be- 
cause the energy of the first excited state of a noble gas is comparable to the ionization energy, and consequently an electron has a high probability of being accelerated to the ionization energy without inelastic collisions. Pure noble gas counters, however, are not stable at high gains, in part because, the gas is transparent to its own characteristic UV radiation, which is emitted during the multiplication process and has sufficient energy to eject electrons from the counter walls and thus re-initiate the pulse process. A quench gas is usually added to absorb the UV radiation; 5-15\% methane or carbon dioxide are common choices. A small quantity of helium also is sometimes included in sealed counters to assist in leak detection. The gas constituents should not include molecules which form negative ions, for the attached electrons are effectively lost since the heavy ions cannot be accelerated to ionization energies between collisions by reasonable electric fields. Finally, the gas X-ray absorption coefficient should not be so high that the desired photons are all absorbed near the counter window, for this results in poor resolution. Electron diffusion is relatively more important in low field regions and consequently there is a larger probability for electron losses to the walls or by recombination. These losses are more severe if a non-conductive window is used, since in this case positive ions created in the multiplication process eventually will collect on the window and cancel the local bias field.
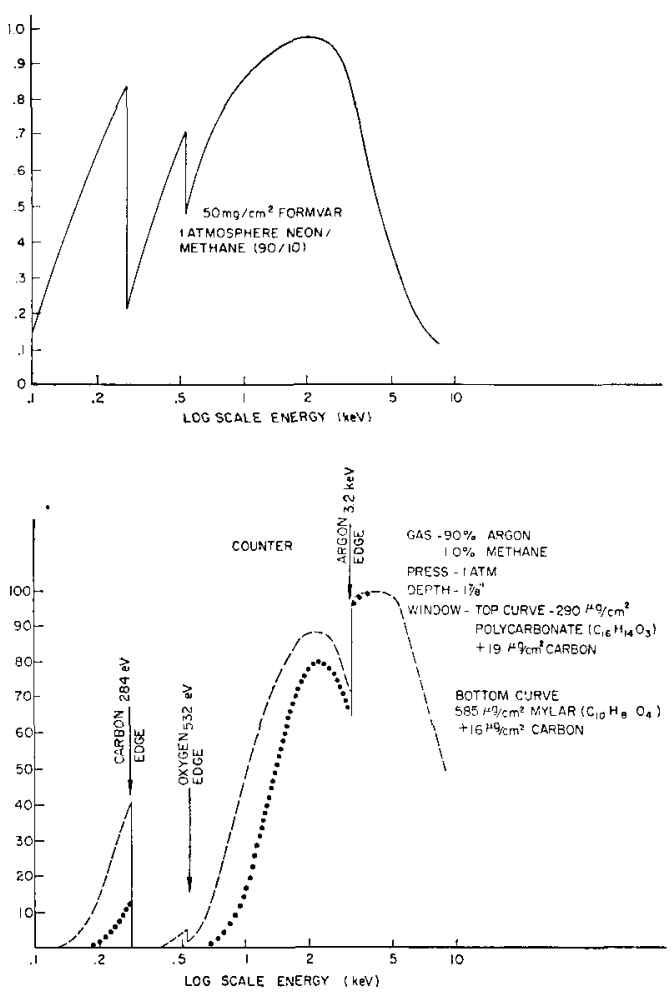

Fig. 2. The quantum efficiencies of proportional counters designed to be sensitive to soft $X$ rays; the counter described in the upper figure was developed by Cornell et al. (1968) of LRL; those in the lower figure by Bunner et al. (1969) of the University of Wisconsin. 
The efficiency of the counter is limited by window absorption at low energy and by gas transmission at high energy. Some representative counter efficiencies are shown in Figure 2; the effects of absorption edges in the counter window and gas are apparent. The efficiency can be extended to higher energies by using a higher atomic number gas such as xenon, but it is difficult to achieve useful efficiencies beyond about $50 \mathrm{keV}$, and $20 \mathrm{keV}$ is a more typical limit.

The resolution of a properly designed counter is limited primarily by the statistical fluctuations in the number of electron-ion pairs formed initially and in the subsequent amplification processes. Charles and Cooke (1968) have performed an extensive study of proportional counter resolution and find that their results are consistent with a gaussian distribution having a variance $\sigma$ given by

$$
(\sigma / E)=[(F+b) \varepsilon / E]^{1 / 2},
$$

where $E$ is the photon energy, $\varepsilon$ is the average energy required to produce an electronion pair, $b$ is a constant between $2 / 3$ and 1 which is taken from the Byrne (1962) model of the fluctuations in the amplification process, and $F$ is the Fano factor which compensates for the fact that the initial ion-electron pairs are not statistically independent events because of energy conservation, but fluctuations in the partition of the initial energy between ionization and other competing channels can still occur. Charles and Cookes' data for P-10 $\left(90 \%\right.$ Argon, $\left.10 \% \mathrm{CH}_{4}\right)$ is consistent with the values $b=0.6$, $F=0.2, \varepsilon=26.5 \mathrm{eV}$, which corresponds to $(\Delta E / E)$ FWHM of 0.35 at $1 \mathrm{keV}$ and 0.145 at $5.9 \mathrm{keV}$. Some values of these parameters for other gases which were calculated or compiled by Alkhazov et al. (1967), are given in Table II.

TABLE II

Proportional counter gas properties ${ }^{\mathrm{a}}$

\begin{tabular}{llll}
\hline Gas & $\varepsilon(\mathrm{eV})$ & F calc. & Fexp. \\
\hline $\mathrm{He}+\mathrm{Ar}$ & 30.1 & 0.055 & \\
$\mathrm{He}+\mathrm{Xe}$ & 28.7 & 0.060 & \\
$\mathrm{He}+\mathrm{CH}_{4}$ & 30.3 & 0.075 & \\
$\mathrm{Ne}+\mathrm{Ar}$ & 25.3 & 0.050 & \\
$\mathrm{Ar}+\mathrm{CH}_{4}$ & 26.0 & 0.17 & 0.19 \\
$\mathrm{Ar}+\mathrm{C}_{2} \mathrm{H}_{2}$ & 20.3 & 0.075 & 0.09 \\
$\mathrm{H}_{2}$ & 36.3 & 0.34 & \\
$\mathrm{~N}_{2}$ & 34.3 & 0.28 & \\
$\mathrm{O}_{2}$ & 30.8 & 0.37 & \\
$\mathrm{CO}_{2}$ & 32.8 & 0.32 & \\
$\mathrm{CH}_{4}$ & 27.3 & 0.26 & \\
$\mathrm{C}_{2} \mathrm{H}_{2}$ & 25.7 & 0.27 & \\
\hline
\end{tabular}

a Alkhazov et al., 1967.

The resolution obtained by Charles and Cooke is somewhat better than typically achieved; they stressed the importance of wire uniformity, and found that a one percent change in wire diameter resulted in a gain change of $8 \%$. The ends of the anode also must be shielded from X-rays because field non-uniformities result in gain shifts 
in these regions. Other effects contributing to poor resolution are relatively unimportant if reasonably pure gasses, well controlled gas densities, and modern charge sensitive electronics are used.

The proportional counter resolution is adequate to determine the general shape of a spectrum and a few spectral parameters, such as the temperature, absorption cutoff energy, and total intensity of a thermal source. It is also possible to detect lines, such as the iron $\mathrm{K}$ emission, which are expected to be isolated. The resolution is not adequate to separate different ionization stages. The resolution also is not adequate for detecting absorption edges, particularly since the most important ones are at lower energies where the resolution is poor.

The sensitivity and effective resolution are usually limited by statistical fluctuations in the background; this is particularly true when the proportional counter is used as the detector for the high resolution but low efficiency crystal spectrometers to be discussed below. The pulse shape discrimination technique, which is very effective in eliminating background events, is based upon the fact that the low energy primary photoelectrons have a small range, whereas a fast particle has a lower ionization cross-section and requires an extended path length to create the same number of electron-ion pairs. In general, the drift times to the anode will vary along the track and the resulting pulse shape will be given by folding the drift time distribution with the pulse shape resulting from a localized charge deposition. This effect was first studied by Mathieson and Stanford (1964) and subsequently by many authors, including Gorenstein and Mickiewicz (1968), Ricke and Gomes (1969), Mathieson and Harris (1970), and Harris and Mathieson (1971). The usual technique is to doubly differentiate the integrated charge pulse; this results in a zero cross over time which is essentially a function of the pulse rise time. The time distribution obtained by Harris and Mathieson for $5.9 \mathrm{keV} \mathrm{X}$ rays and also for background events simulated by $\mathrm{Co}^{60}$ radiation is shown in Figure 3. The

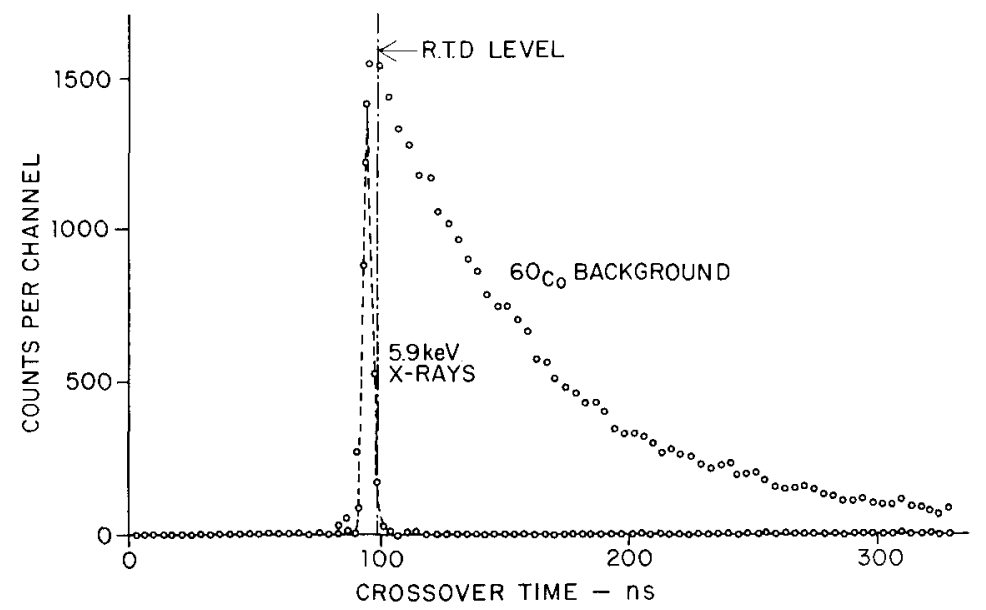

Fig. 3. Pulse shape distributions for $X$ rays and background events obtained by Harris and Mathieson (1971). 


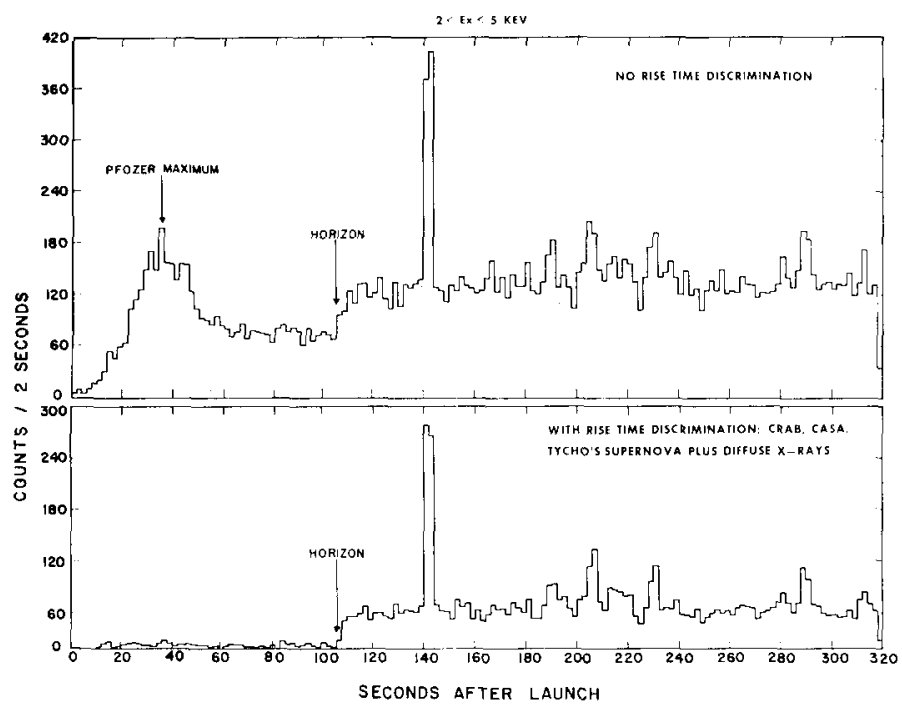

Fig. 4. The effect of the Gorenstein and Mickiewicz (1968) pulse shape discrimination system upon the background encountered in a rocket flight.

background rejection is obviously quite effective. Gorenstein and Mickiewicz (1968) obtained the reduction in the background encountered in a rocket flight which is shown in Figure 4; the events after about $100 \mathrm{~s}$, when the rocket stopped viewing the Earth, are primarily true $\mathrm{X}$ rays from the diffuse background, and the effectiveness of the system is most easily observed in the data obtained before that time.

This background rejection technique is limited at high energies because the photoelectron range becomes large, and at lower energies because the track length of a background event depositing the same energy also must be short. A counter gas with a large probability of de-exciting by X-ray fluorescence rather than by Auger emission will result in a low X-ray detection efficiency; since the gas is relatively transparent to its own fluorescent radiation, this $\mathrm{X}$ ray may be absorbed at some distance from the original site and result in a slower pulse similar to the background events. The loss of the fluorescent photon from the counter results in an energy shift by this amount but no reduction in efficiency due to pulse shape discrimination; this process is familiar to those using proportional counters. The electron diffusion during the time required to drift to the anode ultimately limits the pulse shape discrimination technique; this time is determined by the ratio of the diffusion coefficient to the electron mobility, or the second Townsend coefficient $(\mathrm{K})$. The measurements of this ratio for several gasses by Cottrell and Walker (1961) are reproduced in Figure 5. Our own unpublished data indicate better background rejection with $\mathrm{CO}_{2}$ quench gas than with $\mathrm{CH}_{4}$, which agrees with this analysis. The quench gas must have low energy excited levels, usually rotation or vibration states, in order to reduce the effective electron temperature and obtain a low ratio of diffusion to mobility coefficients. A low molecular weight positive charge carrier is also desirable since this results in a high mobility positive ion and consequently 


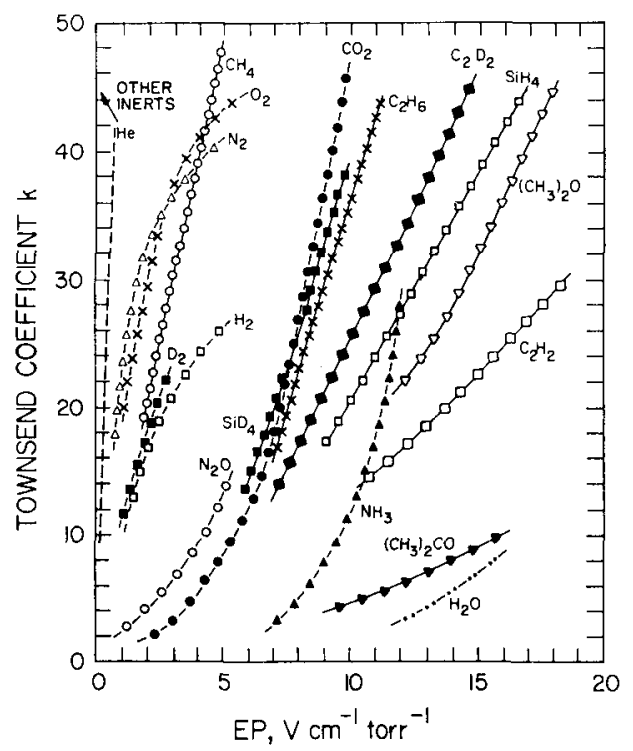

Fig. 5. Measurements of the ratio of electron diffusion coefficient to the electron mobility by Cottrell and Walker (1961).

a faster pulse which preserves the effect of the electron collection time upon the pulse shape. The quench gas often has the lowest ionization potential and becomes the positive charge carrier.

Finally, if an organic quench gas is used, the lifetime of the counter may be limited by the quench gas polymerizing and deposing upon the anode wire. Tananbaum and Kellogg (1970) report that counters with $\mathrm{CO}_{2}$ quench have at least a factor of 10 longer useful life than counters with $\mathrm{CH}_{4}$.

Anti-coincidence counters are most effective if located in the same gas volume as the data counter, but separated from it by cathode wires or screens. This allows the rejection of both penetrating charged particles and also the secondaries from neutrals which interact in the counter wall. Additional data counters may also be used in series, which effectively supplements the intrinsic resolution of the counter with band pass filters; the lower energy $X$ rays are absorbed in the first layer while more penetrating radiation is absorbed in all of the counters. This technique will be used by MIT on OSO-H and SAS-C, by University College London UK-5, and by GSFC on OSO-1 and HEAO-B.

\section{Scintillation Detectors}

The scintillation counter usually is used to determine the shape of a source spectra at higher energies where the efficiency of the proportional counter is inadequate. There are no narrow spectral features expected in this portion of the spectrum, and so relatively crude spectral resolution is sufficient. The energy resolution is determined by the fluctuations in the number of photoelectrons, and thus depends upon the scintillator light 
yield, the light collection efficiency, and the quantum efficiency of the phototube. The energy required per photoelectron is about $250 \mathrm{eV}$ in a system utilizing a thin scintillator with good light collection and a high quantum efficiency phototube. This results in a resolution (FWHM) of about $50 \%$ at $5 \mathrm{keV}$ or $25 \%$ at $20 \mathrm{keV}$. Systems which must be efficient at high energies, and, therefore have thicker scintillators, usually have somewhat poorer resolution.

The principal experimental problem is the elimination of background events. A typical modern counter system developed by the University of California at San Diego group is shown in Figure 6, which was taken from Peterson et al. (1971). This detector

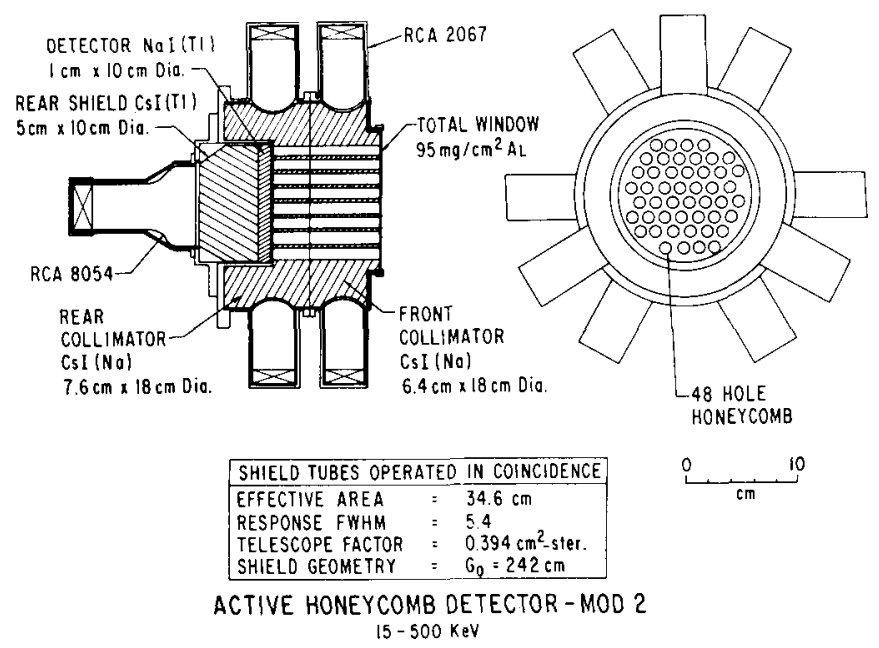

Fig. 6. A modern scintillation detector with active collimator developed by Peterson et al. (1971) of the University of California at San Diego.

utilizes active collimation, which was first developed by Frost et al. (1966) to eliminate background events in which an interaction in the collimator results in a particle entering the scintillator. This particular design also incorporates an active CsI (Tl) shield to eliminate events in which particles or photons enter the $\mathrm{NaI}$ (Tl) from below. The signals from these two channels are distinguished by the different decay times characteristic of the two crystals. The background observed with this detector at balloon altitudes is shown in Figure 7.

\section{Bragg Crystal Spectrometers}

Bragg crystal spectroscopy is the most commonly used technique for obtaining high resolution X-ray measurements, such as the excellent solar data presented at this conference. These instruments are based upon the fact that a crystal will reflect $X$ rays efficiently only if the glancing angle $\theta$ between the incident $X$ rays and the crystal planes approximately satisfies the Bragg condition, $n \lambda=2 d \sin \theta$, where $d$ is the separa- 
tion between reflecting planes and $n$ is the (integer) order of reflection. The useful range of Bragg angles, extends from $\theta \approx 20^{\circ}$ to $\theta \approx 70^{\circ}$. The resolution, which can be obtained by differentiating the Bragg formula, is given by: $(\Delta \lambda / \lambda)=\cot \theta \Delta \theta$. The angular width $\Delta \theta$, over which the amplitudes reflected from the various crystal planes interfere constructively, varies inversely as the depth of penetration of the incident wave into the crystal and consequently is less for higher energies. The penetration depth usually is limited by the coherent reflection rather than by absorption at higher X-ray energies.

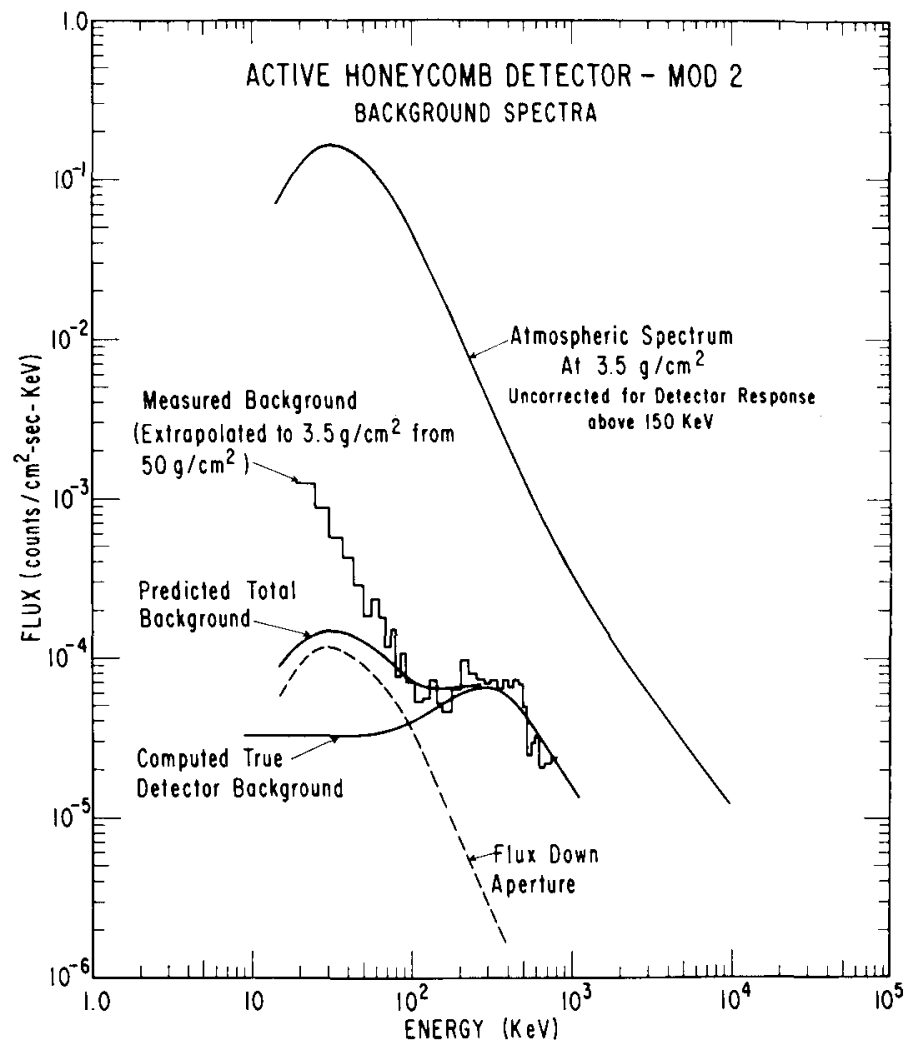

Fig. 7. The background observed at balloon altitudes with the detector shown in Figure 6.

Measurements are normally made by varying the crystal angle, or 'rocking', over a small angular range about the Bragg angle corresponding to the wavelength of the expected feature. This procedure requires less precise pointing and alignment than attempting to perform measurements at the peak of the reflection curve, and also provides an estimate of the background and source continuum contributions which must be known to evaluate the data. The total number of signal counts from a line will be proportional to the integral of the reflection probability, which is usually expressed in radians. It is useful to make these efficiency and resolution definitions more specific; let 
the reflection probability for $\mathrm{X}$ rays of wavelength $\lambda$ incident upon a crystal at glancing angle $\theta$ be $P(\theta, \lambda)$. Then the integrated reflection coefficient is defined as

$$
R_{\mathrm{c}}(\lambda)=\int \mathrm{d} \theta P(\theta, \lambda)
$$

we denote the FWHM of the curve as $\Delta \theta$ and the peak reflectivity as $P_{0}$. Absolute measurements of single crystal parameters are difficult in practice because of the problems in obtaining an intense, spectrally pure, and stable beam with a sufficiently small angular divergance, and only isolated measurements at wavelengths of interest to X-ray astronomy appear in the literature. The properties of a particular crystal also depend upon its history. A freshly cleaved or properly polished and etched crystal will reflect essentially as single crystal, whereas a crystal with an abraded surface will act as a mosaic of small perfect crystals which are slightly mis-aligned with respect to one another. The reflection probability for such a small crystal varies as the square of the number of scattering centers, and, therefore, is enhanced compared to the absorption which varies only linearly; consequently, there is a reasonable probability that an $\mathbf{X}$ ray having a slightly incorrect angle will penetrate through the small crystals until it strikes one which is properly oriented for reflection. The net result, if absorption is not dominant, is that the integrated reflectivity and the width of the reflection curve are both larger for mosaic crystals than for perfect crystals, but the peak reflectivity is usually less. Novick and his colleagues at Columbia have developed mosaic crystals, particularly of graphite and $\mathrm{WS}_{2}$, which have approximately the theoretical performance.

TABLE III

Typical crystal properties

\begin{tabular}{|c|c|c|c|c|}
\hline Crystal & Formula & $2 d(\AA)$ & $\begin{array}{l}\text { Resolution } \\
(\lambda \Delta \lambda)\end{array}$ & $\begin{array}{l}R_{\mathbf{c}} \\
\text { (Radians) at } \lambda(\AA)\end{array}$ \\
\hline $\mathrm{LiF}[1]$ & $\mathrm{LiF}$ & 4.206 & 1000 & $0.7 \times 10^{-4} 2.1$ \\
\hline Calcite[2] & & 6.06 & 10000 & $3 \times 10^{-5} \quad 3$ \\
\hline Germanium[2] & $\mathrm{Ge}$ & 6.55 & 10000 & $3 \times 10^{-5} \quad 3$ \\
\hline PET[3] & $\mathrm{C}_{5} \mathrm{H}_{12} \mathrm{O}_{4}$ & 8.75 & 600 & $10^{-4} \quad 1.5$ \\
\hline EDDT[3] & $\mathrm{C}_{6} \mathrm{H}_{14} \mathrm{O}_{6} \mathrm{~N}_{2}$ & 8.80 & 1000 & $8 \times 10^{-5} \quad 1.5$ \\
\hline $\mathrm{ADP}[2]$ & $\mathrm{NH}_{4} \mathrm{H}_{2} \mathrm{PO}_{4}$ & 10.64 & 5000 & $10^{-5} 8$ \\
\hline Gypsum & $\mathrm{CaSO}_{4}$ & 15.2 & & \\
\hline KAP[2] & $\mathrm{C}_{8} \mathrm{H}_{5} \mathrm{O}_{4} \mathrm{~K}$ & 26.63 & $\begin{array}{r}2000 \\
500\end{array}$ & $\begin{array}{ll}6 \times 10^{-5} & 10 \\
6 \times 10^{-5} & 10\end{array}$ \\
\hline $\mathrm{OHM}[2]$ & $\mathrm{C}_{22} \mathrm{H}_{40} \mathrm{O}_{4}$ & 63.65 & $\sim 400$ & $10^{-5} \quad 55$ \\
\hline Graphite[4] & ${ }^{* *} \mathrm{C}$ & 6.7 & $\sim 70$ & $1.0 \times 10^{-3} \quad 4.73$ \\
\hline Myristate & $\mathrm{x}\left(\mathrm{C}_{14} \mathrm{H}_{27} \mathrm{O}_{2}\right)[2]$ & 79 & $\sim 100$ & \\
\hline Sterates & $\mathrm{x}\left(\mathrm{C}_{18} \mathrm{H}_{55} \mathrm{O}_{2}\right)[2]$ & 100 & $\sim 100$ & \\
\hline
\end{tabular}

** MOSAIC

$x$ Bivalent Cation ( $\mathrm{Ba}, \mathrm{Pb}$, etc.)

[1] Vierling et al. (1969).

[2] Walker and Rugge (1971).

[3] Ruderman and Michelman (1968)

[4] Novick (1971). 
Some properties of crystals which are useful for X-ray astronomy are given in Table III. Several groups, including Pounds and co-workers at the University of Leicester, Walker at Aerospace, Acton at Lockheed, and Novick, Angel, and coworkers at Columbia are now measuring single crystal properties, and hopefully more complete data will be available soon. The measurements of KAP properties by

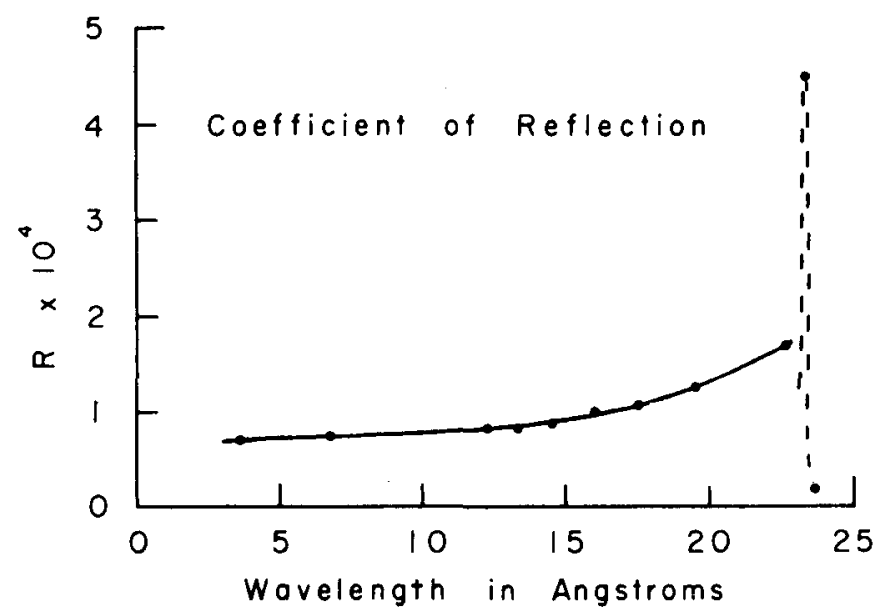

Fig. 8. The two crystal integrated coefficients of reflection, $R_{\mathrm{c}}$, of KAP crystals measured by Liefeld et al. (1970).

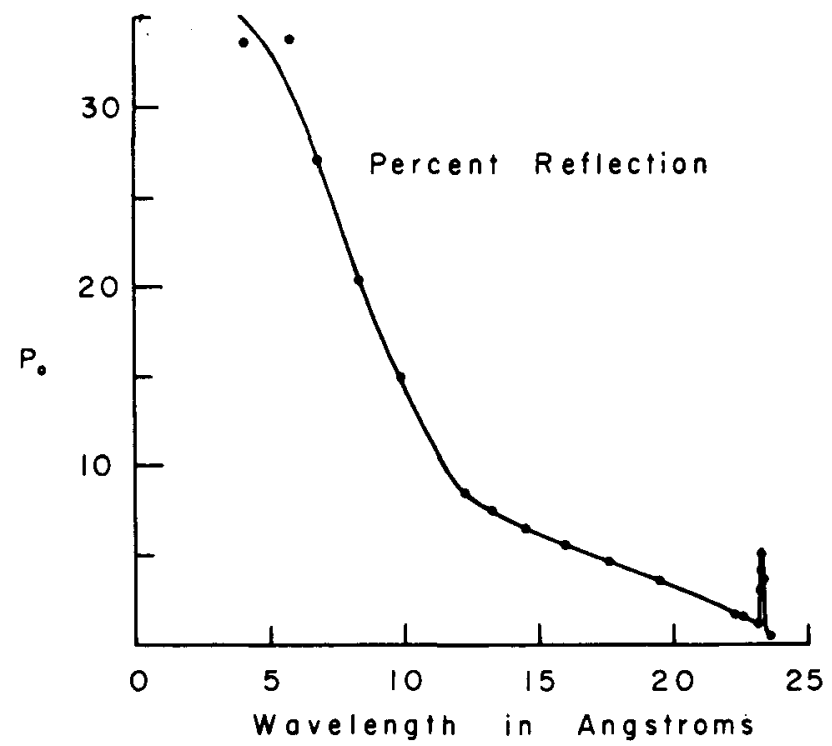

Fig. 9. The peak reflectivity of KAP crystals measured with a two crystal apparatus by Liefeld et al. (1970). 
Liefeld et al. (1970) are summarized in Figures 8, 9, and 10; these are two crystal measurements in which a similar crystal is used to obtain a collimated, monochromatic beam. The numerical factors required to convert this data to single crystal properties are of order unity but depend upon the angle and polarization and are difficult to calculate when absorption is important. These measurements can be used to estimate ex-

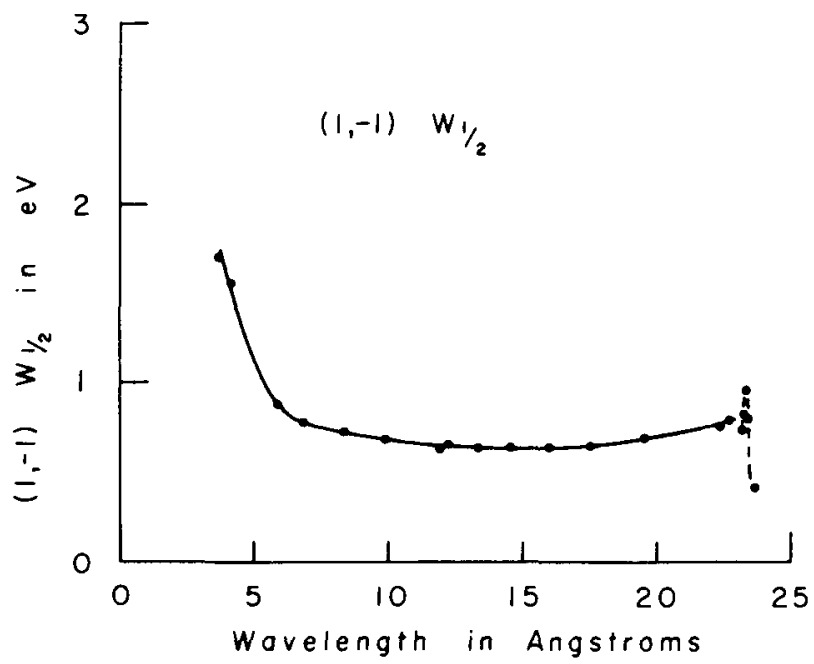

Fig. 10. The energy widths at half maximum of the first order parallel position rocking curves of KAP crystals measured by Liefeld et al. (1970).

perimental sensitivity, but should not be used in the analysis of data because of these difficulties and the variations from crystal to crystal. The most striking features of these curves are the relatively constant values of the integrated reflectivity and the width of the reflection curve when expressed in energy units. The anomalous behavior associated with the oxygen edge is quite apparent.

Precise absolute wavelength measurements require corrections for the index of refraction in the crystal. Blake (1970) has calculated this effect for KAP and finds excellent agreement with oxygen Lyman- $\alpha$ and $-\beta$ measurements; he expresses his results as corrections to the crystal grating constant.

We now consider measurements of a spectral line of intensity $I_{L}$ photons $\mathrm{cm}^{-2} \mathrm{~s}$ superimposed upon a continuum flux of $I_{\mathrm{c}}(\lambda)$ photons $\mathrm{cm}^{-2} \mathrm{~s} \AA$. We also assume a collecting area $\mathrm{A}$, a detector area $\mathrm{A}_{\mathrm{D}}$, detector efficiency $\varepsilon$, and a background, $\mathrm{B}_{\mathrm{D}}$ proportional to the area of the detector. The contribution of the diffuse X-ray background is assumed to be negligible. We assume that a uniform scan through an angular range $\Phi$ is performed in a time $t$. Then, the number of signal counts will be $N_{\mathrm{L}}$ given by:

$$
N_{\mathrm{L}}=\varepsilon t \mathrm{~A} I_{\mathrm{L}}\left(R_{\mathrm{c}} / \Phi\right)
$$


the number of counts contributed by the source continuum in the same angular interval as the line will be $N_{\mathrm{c}}$, given by

$$
\begin{aligned}
N_{\mathrm{c}} \approx \frac{\varepsilon \mathrm{A} t}{\Phi} \int_{\theta_{0}-(\Delta \theta / 2)}^{\theta_{0}+(\Delta \theta / 2)} \mathrm{d} \theta \mathrm{d} \lambda I_{\mathrm{c}}(\lambda) & P(\theta, \hat{\lambda}) \approx \\
& \approx \varepsilon \mathrm{A} t_{\mathrm{c}}\left(\begin{array}{l}
2 d \\
n \\
\cos \theta_{0}
\end{array}\right) \frac{\Delta \theta R_{\mathrm{c}}}{\Phi} \approx \varepsilon \mathrm{A} t\left(I_{\mathrm{c}} \Delta \lambda\right) \frac{R_{\mathrm{c}}}{\Phi}
\end{aligned}
$$

In this expression, $\theta_{0}$ is the Bragg angle for the line wavelength, we have assumed that $I_{c}$ varies slowly in the region of the line contribution, and we have performed the following substitution

$$
\int \mathrm{d} \lambda P(\theta, \lambda) \approx \int \mathrm{d} \lambda P\left(\theta-\theta_{0}(\lambda)\right) \approx \int \mathrm{d} \theta_{0} \frac{\mathrm{d} \lambda}{\mathrm{d} \theta_{0}} P\left(\theta-\theta_{0}\right) \approx R_{\mathrm{c}}\left(\mathrm{d} \lambda / \mathrm{d} \theta_{0}\right) .
$$

The number of background counts is given by

$$
N_{\mathrm{B}}=\mathrm{B}_{\mathrm{D}} \mathrm{A}_{\mathrm{D}} t(\Delta \theta / \Phi)
$$

where the background term $B_{D}$ includes only those events satisfying the X-ray criteria, which may also include crude energy selection, and we have neglected the small contribution of the diffuse X-ray background.

Consider, for example, a rocket flight dedicated to observing the $\mathrm{Ne} x$ line at $1.02 \mathrm{keV}$, which is the strongest line predicted in the Tucker (1967) model of Sco X-1. The sensitivity is determined essentially by the fluctuations in $N_{c}$ and the detector background; we choose the following values which are typical for a rocket with reasonable pointing limitations and a proportional counter with good background rejection; $A=\mathrm{A}_{D}=$ $500 \mathrm{~cm}, t=200 \mathrm{~s}, \Phi=3 \times 10^{-2}$ radians, and $\mathrm{B}_{\mathrm{D}}=2 \times 10^{-3} \mathrm{~cm}^{-2} \mathrm{~s}^{-1}$. We also assume a KAP crystal with a reflection efficiency of $6 \times 10^{-5}$ radians and an energy resolution of $0.67 \mathrm{eV}$. We obtain $N_{\mathrm{c}} \approx 7, N_{\mathrm{B}} \approx 2$, and the minimum detectable line strength must result in about $3 \sqrt{ } 9$ or approximately 9 counts. This corresponds to $I_{L} \approx 0.05$ photons $\mathrm{cm}^{-2} \mathrm{~s}^{-1}$, or about $1 / 20$ of the Tucker predicted line strength. This, however, is extremely optimistic since we have assumed no line broadening due to electron scattering, no interstellar absorption, and an entire rocket flight devoted to one feature in the strongest $\mathrm{X}$-ray source. We conclude that X-ray crystal spectroscopy experiments on sounding rockets are at best limited to the very strongest $\mathrm{X}$-ray sources.

A similar experiment flown in a satellite is more promising. Let us assume, for example, that the area can be increased to $2000 \mathrm{~cm}^{2}$ and the time to $5 \times 10^{4} \mathrm{~s}$, or about one day's observation with allowance for occultations. Then the same pointing capability would allow us to detect the Tucker predicted line strength in a source of about $10^{-3} \mathrm{Sco}-\mathrm{XI}$. If the satellite were spinning, the sensitivity during the same period would be reduced to about $10^{-2}$ Sco-XI. These numbers are limited by the counter background rather than by the source continuum, and so depend directly upon the 
line strength; for example, if the intensity in a line is reduced by a factor of ten due to electron scattering or other causes, then the detectable source strengths are ten times larger than given here. It is likely that even satellite experiments will be limited to the stronger sources.

For very strong sources we can hope to perform Doppler shift and line broadening measurements as well. We can restate the expected experimental errors as follows:

$$
\frac{\sigma_{I}}{I} \approx \frac{\left(\Phi / R_{\mathrm{c}}\right)^{1 / 2}}{I_{\mathrm{L}}(\varepsilon \mathrm{A} t)^{1 / 2}}\left[\mathrm{~B}_{\mathrm{D}}\left(\frac{\Delta \theta}{\Phi}\right)\left(\frac{\Phi}{R_{\mathrm{c}}}\right)\left(\frac{\mathrm{A}_{\mathrm{D}}}{\varepsilon \mathrm{A}}\right)+\left(I_{\mathrm{c}} \Delta \lambda\right)+I_{\mathrm{L}}\right]^{1 / 2} .
$$

Wavelength centroid statistical limit

$$
\sigma_{\lambda} \approx\left(\begin{array}{c}
\sigma_{I} \\
I
\end{array}\right) \frac{\Delta \lambda}{2 \sqrt{2 \ln 2}}
$$

Line broadening statistical limit, or variance of $(\Delta \lambda)^{2}$.

$$
\sigma(\Delta \lambda)^{2} \approx 2\left(\sigma_{I} / I\right)(\Delta \lambda)^{2}
$$

Small factors depending upon the shape of the crystal reflection curves are neglected in all of the above formulae. We also assume that the crystal width and the line width add quadradically to result in $(\Delta \theta)^{2}$ or $(\Delta \lambda)^{2}$. If we assume the same experiment parameters, and $I_{L}=0.1$ photon $\mathrm{cm}^{-2} \mathrm{~s} \approx 10^{-1} \mathrm{Sco}-\mathrm{XI}$, we find that $\left(\sigma_{I} / I\right) \approx 7 \times 10^{-3}$. This corresponds to an error in Doppler shift measurements of $(\delta \lambda / \lambda)={ }^{\prime} 2 \times 10^{-6}$ if statistics were the only limitation. In this case the accuracy of the source position and vehicle aspect data would probably limit the accuracy of the Doppler measurement to $(\delta \lambda / \lambda) \approx 10^{-4}$ or $30 \mathrm{~km} \mathrm{~s}$. The detectable source broadening, again using the $3 \sigma$ criterion, would have a statistical limit of $(\delta \lambda / \lambda) \approx 2 \times 10^{-4}$; this corresponds to the thermal Doppler broadening at about $1.6 \times 10^{6} \mathrm{deg}$.

To summarize these results, the sensitivity of crystal spectrometers is not adequate for rocket measurements of most sources. In satellites it should be possible to detect lines for sources of moderate intensity $\left(\sim 10^{-3} \times\right.$ Sco-XI model $)$ and to determine meaningful line intensity ratios for somewhat stronger sources. In these cases it will be possible to determine the same source characteristics as have been presented for solar features at this conference, that is composition, temperature by determining the ionization equilibrium, and density by measuring the He I like ratios and using the Gabriel and Jordan (1969) analysis. The Doppler shifts of these lines can be determined to about $30 \mathrm{~km} \mathrm{~s}^{-1}$. The strong $\left(\sim 10^{-1} \mathrm{Sco}-\mathrm{XI}\right.$ model $)$ sources will allow more meaningful Doppler velocity measurements if the vehicle aspect and source positions are known. Line broadening for reasonable temperatures will also be measurable for these sources. This may be consistent with that predicted from the ionization equilibrium measurements, or it may be larger and indicate large mass motions, such as would be caused by turbulence.

There are a number of such satellite experiments now being designed or constructed; these include the Columbia OSO-1 experiment, which employs mosaic graphite and 
utilizes the vehicle rotation to scan the spectrum; the University of Leicester experiment on UK-5; the MIT-AS \& E experiment on the ANS satellite; and the WalkerAngel experiment on HEAO-B. The latter, which will be the largest and most sensitive of these instruments, will have three crystals, each with an effective area of about $2500 \mathrm{~cm}^{2}$.

\section{Focusing Instruments}

The sensitivity of all of these instruments is limited by the detector background and, can be improved by using focusing techniques to reduce the area of the detector and the detector noise. Giacconi and Rossi (1960) first suggested the use of focusing X-ray telescopes for X-ray astronomy two years before the discovery of the first X-ray source. The most frequently used design consists of concentric confocal surfaces generated with conic sections, as illustrated in Figure 11. These telescopes were first analyzed by

a.

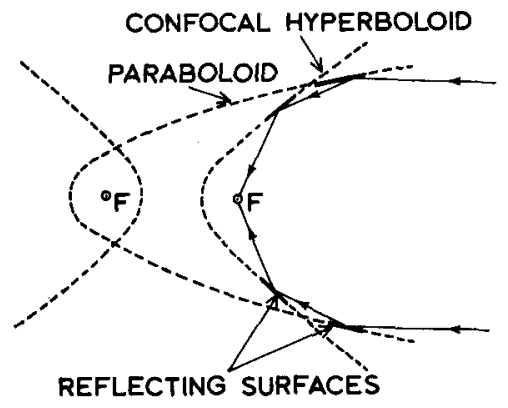

b.

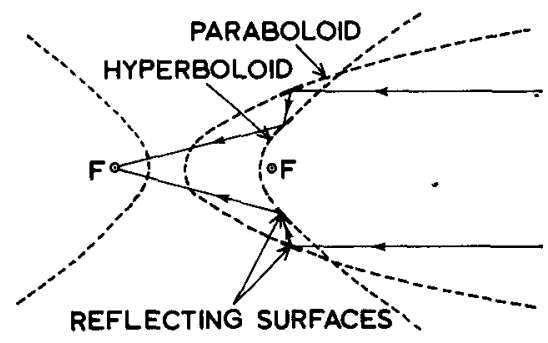

c.

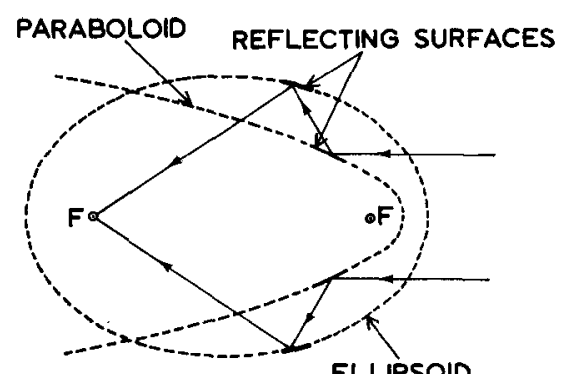

Fig. 11. Three possible configurations for image forming X-ray telescopes consisting of concentric, confocal surfaces of revolution generated by conic sections. 
Wolter (1952a, b) and more recently by Van Speybroeck and Chase (1971). The progress in fabricating these mirrors was reviewed by Giacconi et al. (1969). The efficiency of the telescope depends upon the grazing angle, the surface material, and the surface finish, and theoretically approaches the geometrical area, except for narrow absorption edge effects, for all wavelengths longer than some cutoff where the efficiency drops rapidly. The resolution usually is limited by the scatter from the surface imperfections rather than by the optical design; this results in a highly peaked distribution but with significant wings which contain a large fraction of the transmitted energy. The best polished flats yield an approximately gaussian scattering distribution with a standard deviation of about $4^{\prime \prime}$ when $8.3 \AA$ radiation is reflected at a grazing angle of $1^{\circ}$. The point source distribution of a telescope having this surface quality would contain onehalf of the transmitted power in a diameter of about $5^{\prime \prime}$, which may be considered to be the useful resolution for spectroscopic purposes.

\section{Focusing Crystal Spectrometers}

Crystal spectrometers employing point to point or point to line focusing are commonly used in the laboratory and can be adapted to the telescope geometry as shown in Fig. ure 12. In this geometry the source image and detector or appropriate apertures are located on the Rowland circle along with a crystal which is tangent to the Rowland circle

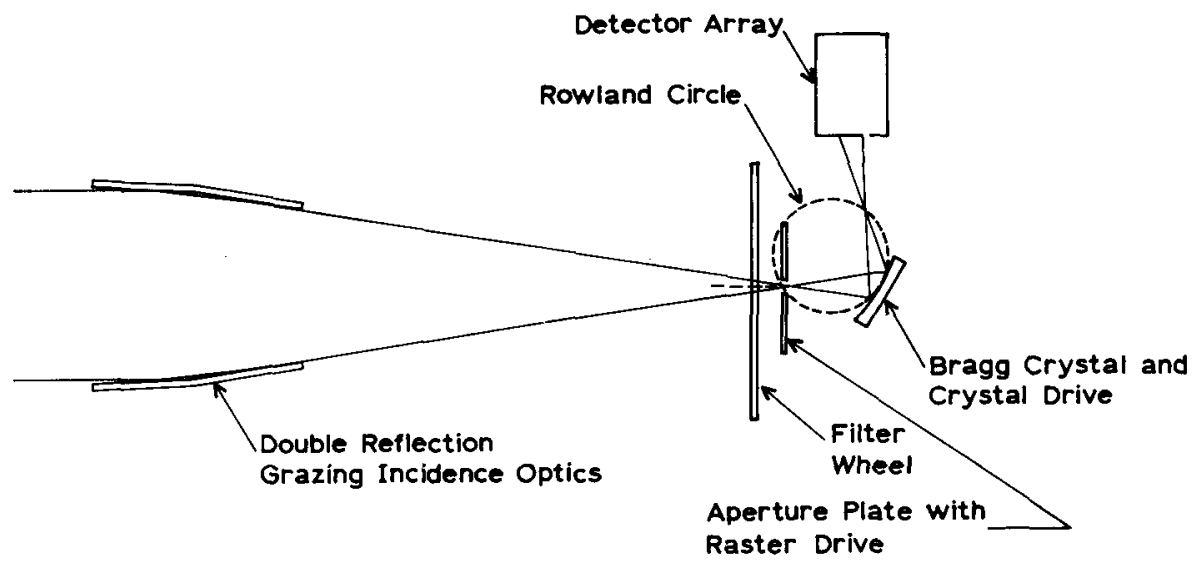

Fig. 12. A spectrometer concept utilizing the Rowland circle geometry and an X-ray telescope.

and has twice its radius of curvature. All rays strike the crystal surface at approximately the same angle and so only a narrow wavelength range will be reflected efficiently. If the crystal is cylindrically bent the resulting image will be a line, while spherical bending will result in a point image. The telescope effective resolution must be multiplied by the ratio of the telescope and crystal focal lengths to determine the degradation in resolution due to this effect. This factor is usually about 10 , and so the effective source size in about one arc minute, which is comparable to or less than the re- 
solution of most crystals used for soft $\mathrm{X}$ rays. A system of this type will be flown as part of the AS \& E, Columbia, GSFC, MIT experiment on HEAO-C. In this case, the effective collecting area will be about $250 \mathrm{~cm}^{2}$, but the detector area will be reduced to about $0.3 \mathrm{~cm}^{2}$ for a line focus, or about $2 \times 10^{-4} \mathrm{~cm}^{2}$ for a point focus. Schnopper and Kaletta (1969) have proposed a variation of this geometry in which the detector is brought closer to the crystal. This results in better resolution because the effects of aberations due to the angular divergence of the beam and crystal bending imperfections are reduced; however, the sensitivity is poorer because a larger detector area must be used.

Angel and Weisskopf (1970) have proposed an objective crystal spectrometer utilizing mosaic crystals and a focussing collector; the concept is illustrated in Figure 13.

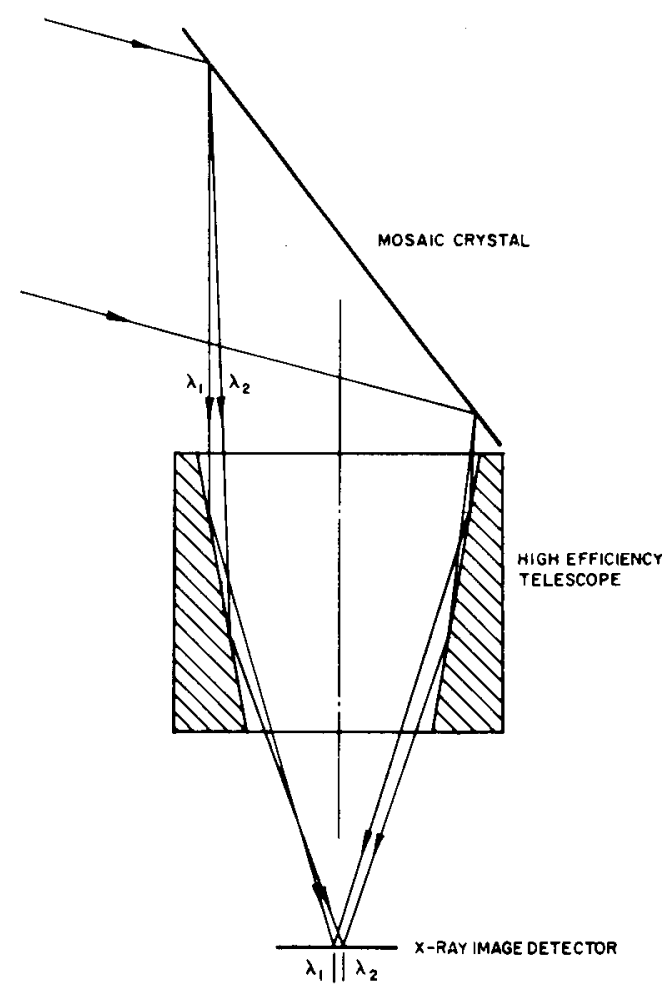

Fig. 13. An objective crystal spectrometer proposed by Angel and Weisskopf (1970). Each wavelength is reflected into a specific cone by the mosaic crystal, and then imaged into an arc by the telescope.

The angle between an incident ray and the ray reflected by a mosaic crystal depends only upon the wavelength, since each photon only will be reflected by a properly oriented subcrystal; the actual angular width will depend upon the size of the subcrystal and will be comparable to the angular width of the perfect crystal reflection curve. A single wavelength from a point source is thus reflected into a cone and will be 
imaged by a telescope into an arc corresponding to its Bragg angle. This instrument obtains the large integrated efficiency of the Mosaic crystal without sacrificing resolution; furthermore, a relatively small detector area can be used because of the telescope focussing. At present this technique is limited to energies above about $1 \mathrm{keV}$ because absorption makes mosaic crystals impractical for softer radiation.

\section{Grating Spectrometers}

Reflection gratings also can be used effectively at X-ray wavelengths. The usual arrangement is similar to the Rowland circle geometry illustrated in Figure 12, with the grating in place of the crystal, but with much smaller grazing angles to obtain useful reflection efficiencies. The gratings reflect all wavelengths simultaneously, each wavelength being focussed to a different spot along the Rowland circle. The disadvantages are the difficulty of obtaining large areas, and the inefficient match between the cone angle from a typical telescope and the angular range for which the grating will reflect efficiently. The mis-match can be minimized by using the type two telescope geometry (Figure 11) which results in a smaller cone angle, and also a larger focal plane scale than would result from a simple lens placed at the mirror position. The Neupert and Underwood OSO-H solar EUV instrument utilizes this telescope and grating configuration.

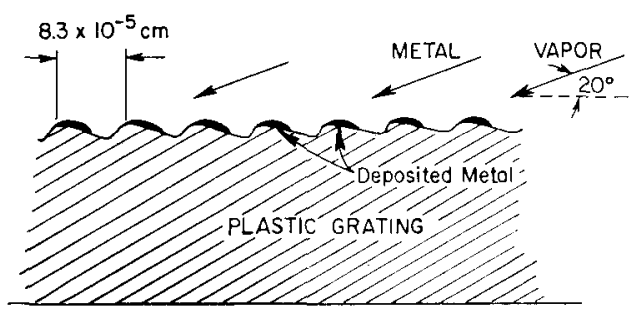

Fig. 14. Transmission grating fabrication technique suggested by Gursky and Zehnpfennig (1966).

R. Speer, J. M. Bennett, and D. Turner of Imperial College, London and A. Franks of NPL, have developed laminar or phase gratings for use in the 1-300 $\AA$ interval. The cross section of the grating surface is similar to a square wave, with the depth of the step chosen to maximize the efficiency for a selected wavelength and angle of incidence; this is approximately the step height which causes the amplitudes reflected from the two surfaces to interfere destructively in the zero order. A typical grating is used at a grazing angle of $1.8^{\circ}$, has 300 lines $\mathrm{mm}$, steps of about $110 \AA$ depth, and is gold coated. They have achieved over $10 \%$ efficiency in each first order reflection for $8.3 \AA$ $\mathrm{X}$ rays with a grating of this type. This performance indicates that excellent surface finish has been achieved in both levels of the grating. The resolution is limited by slit widths and mechanical imperfections rather than by the number of lines; Speer et al. (1970) have achieved resolutions $(\lambda / \Delta \lambda)$ between about 100 and 500 in the range from 1.5 to $8.3 \AA$. 
Gursky and Zehnpfening (1966) suggested an objective grating spectrometer consisting of transmission gratings and a telescope. The grating is placed near the mirror, and the resulting point source focal plane image is a zero order point containing all wavelengths and a line image dispersed according to the normal incidence grating formula $\theta=n \lambda / d$, where $d$ is the grating constant. The spectra of all sources in the field of view are obtained simultaneously; this yields greater efficiency for isolated sources, but can cause a loss of information when an extended object is observed.

The technique for fabricating these gratings is illustrated in Figure 14; thin absorbing lines are formed by evaporating gold onto a thin plastic replica grating at an angle such that shadowing occurs. The efficiency is limited at long wavelengths because of substrate opacity, and at short wavelengths because of the gold becoming transparent. Most of our present gratings are made with a one micron parylene replica grating having 1400 lines per $\mathrm{mm}$; we have used up to 3600 lines $\mathrm{mm}^{-1}$, but the resulting thickness of absorbing gold lines is not adequate for the shorter wavelengths. The resolution of the spectrometer is determined by the telescope rather than the grating; a 1400 line $\mathrm{mm}$ grating results in $0.17 \AA$ resolution in first order with a five arc second resolution telescope. The Tungsten $M$ lines at 6.74 and $6.93 \AA$ were easily separated in the laboratory. Electron microscope photographs of a cross-section of a typical grating show that the gold lines are not symmetric about a normal to the surface; this results in a left-right asymmetry due to the refraction of the amplitude transmitted through the gold; this asymmetry also is observed. Our present gratings have efficiencies of about $9 \%$ in first order at $8.3 \AA$, and are useful in the wavelength ranges 6 to $25 \AA$ and also 44 to $60 \AA$.

A detector which is sensitive to an extended field without loss of spatial resolution is required to effectively utilize the objective grating spectrometer; this is also valuable for imaging experiments in which the source is extended, has an unknown position, or must be viewed from a vehicle with only moderate pointing capabilities. The channel plate multiplier is suitable for these purposes. The device consists of an array of many small parallel tubes of glass; an electric field is maintained along the length of the tubes by applying a potential of about one kilovolt across the array. An X-ray photon striking the tube walls near the front will produce photoelectrons which will be accelerated down the channel; these higher energy electrons will strike the tube walls and produce additional secondary electrons; an electron gain of a few thousand is readily achieved. After the electrons leave the individual tubes they strike a phosphor screen located nearby at a positive potential of several thousand volts with respect to the channel plate. The light produced in the phosphor screen is collected by a relay lens system and imaged onto a standard video tube. Parkes et al. (1970b); have developed an alternative readout system in which the output charge is collected by a grid of wires which are capacitively coupled together; the position is then determined by measuring the charge division. They detected only one position coordinate, but two wire grids at right angles can be used to obtain two dimensional positions. The voltages and grid openings would be adjusted to obtain approximately equal charges upon both grids. 


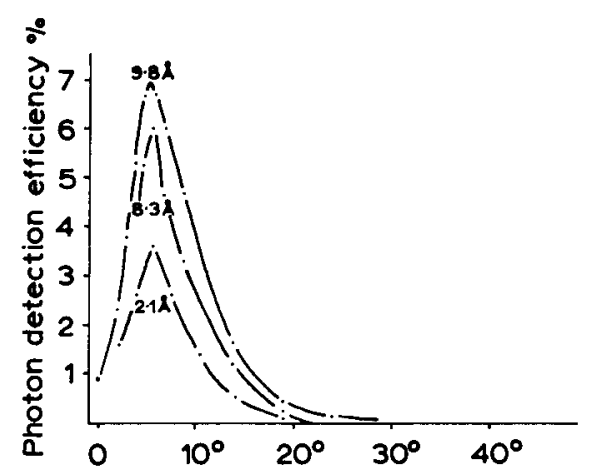

(A) Angle of incidence to channels

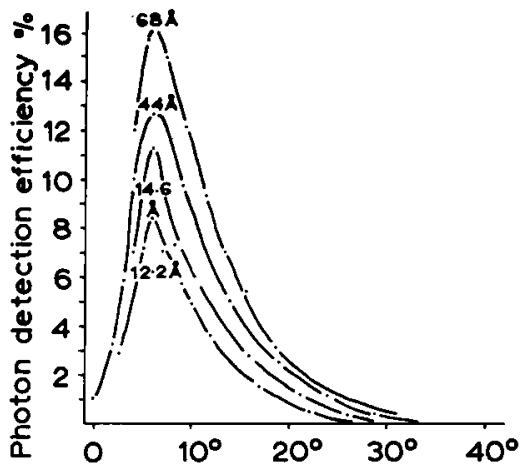

(B) Angle of incidence to channels

Fig. 15. The quantum efficiency of channel plate multipliers for various wavelengths and incident angles. (Parkes et al., 1970a).

The quantum efficiency obtained by Parkes et al. (1970a) is shown in Figure 15. Similar measurements performed in our laboratory agree with these results within experimental errors. The noise rates we have observed for a clean, properly mounted unit are typically a few counts per second to less than one per second over the entire face of the device. There is some hope that the quantum efficiency eventually can be improved by depositing a more efficient photocathode material into the tubes. The quantum efficiency of channeltrons coated with $\mathrm{Mg} \mathrm{F}_{2}$ was measured by Smith and Pounds (1968) and is shown in Figure 16; these values were observed after allowing sufficient time for quantum efficiency to stabilize.

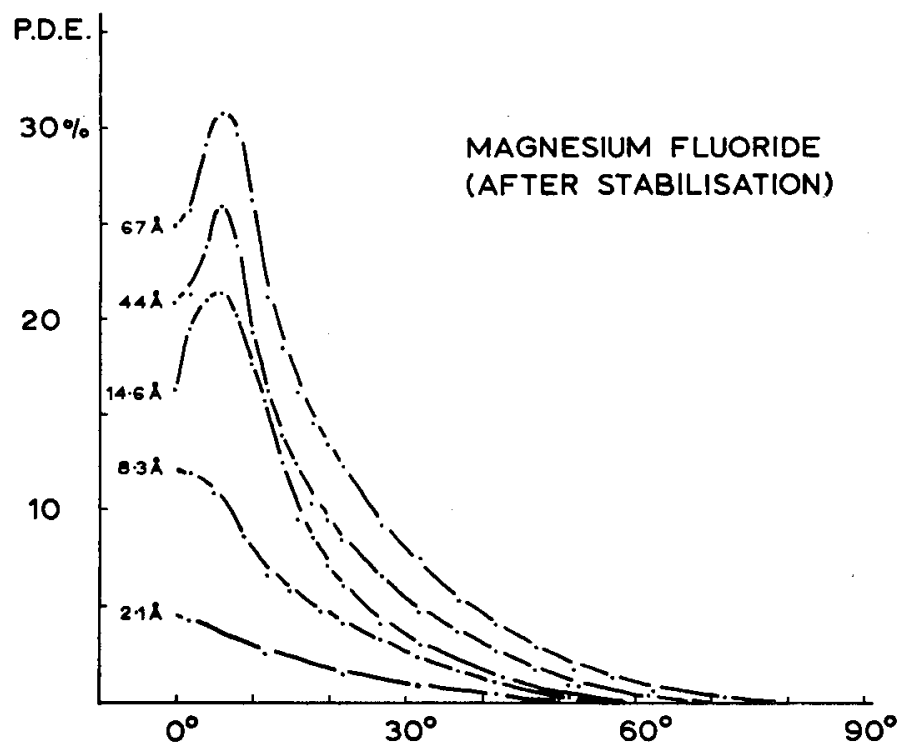

Fig. 16. The quantum efficiency of a channel multiplier coated with $\mathrm{Mg} \mathrm{F}_{2}$ after stabilization. The measurements were performed by Smith and Pounds (1968). 


\section{Solid State Detectors}

The X-ray telescope also makes the use of small, low noise solid state detectors practical. These devices are essentially diodes operated with a reverse bias voltage; an $X$ ray photon absorbed in the depletion layer results in electron hole pairs which are collected without gain by the external circuitry. The detector and preamplifier must be cooled to liquid nitrogen temperatures to achieve satisfactory performance. Either silicon ( $\mathrm{Li}$ ) or germanium ( $\mathrm{Li}$ ) detectors can be used; the germanium detectors require about $3 \mathrm{eV}$ per electron hole pair compared to about $3.8 \mathrm{eV}$ for the silicon detectors and, therefore, potentially have better resolution; however, the silicon is less absorptive so that the effect of the lead layer at the entrance window is less, and the silicon detectors can be stored at room temperatures without rapid deteriotation, whereas the germanium detectors must be kept cold at essentially all times. These factors make the silicon detectors more useful for soft X-ray detection at this time.

The detector efficiency is determined by the entrance electrode and subsequent dead layer. Jaklevis and Goulding (1971) used an entrance electrode of $200 \AA$ of gold and observed a lead layer of $0.2 \mu$ of Si at a bias of $500 \mathrm{~V}$. Their calculated efficiencies as a function of wavelength are reproduced in Figure 17.

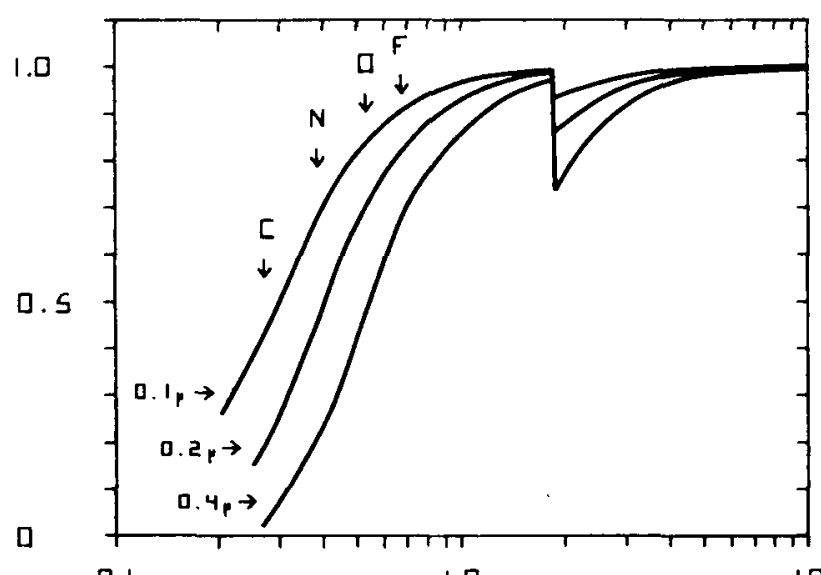

0. 1

1.0

10

PHOTON ENERGY [KEV]

Fig. 17. Solid state detector efficiencies for different silicon dead layer thicknesses. The window also includes a $200 \AA$ gold electrode. (Jaklevis and Goulding, 1971).

There are three pricipal contributions to the resolution; the preamplifier noise, the statistical fluctuations in the number of initial electron hole pairs, and the fluctuations in the efficiency of charge collection. The latter is proportional to $(1 / \mathrm{V} \text { bias })^{2}$ and can be made negligible. The fluctations in electron-ion pairs have the same form given earlier for the proportional counter but with the constant $b$ equal to 
zero. Zulliger and Aitken (1970) find a Fano factor of about 0.13 in practice and suggest a theoretical limit of 0.05 . The preamplifier noise is the dominant resolution factor for low energy X rays; Frankel and Aitken (1970) report a preamplifier noise of $90 \mathrm{eV}$, and Jaklevis and Goulding observed $118 \mathrm{eV}$. The latter authors obtained a resolution (FWHM) of $126 \mathrm{eV}$ for oxygen $\mathrm{K}(525 \mathrm{eV})$ and $137 \mathrm{eV}$ for aluminum $\mathrm{K}$ $(1480 \mathrm{eV})$ radiation. The preamplifier noise varies with the capacitance, and, therefore with the size of the detector and the bias voltage. The effect of detector area upon resolution can be seen in Figure 18.

The resolution of the detector is sufficient to resolve the Lyman- $\alpha$ lines of all elements heavier than oxygen, and is more than adequate for determining the spectral shape of a source. It's unique value, however, is its sensitivity for broad lines and the source

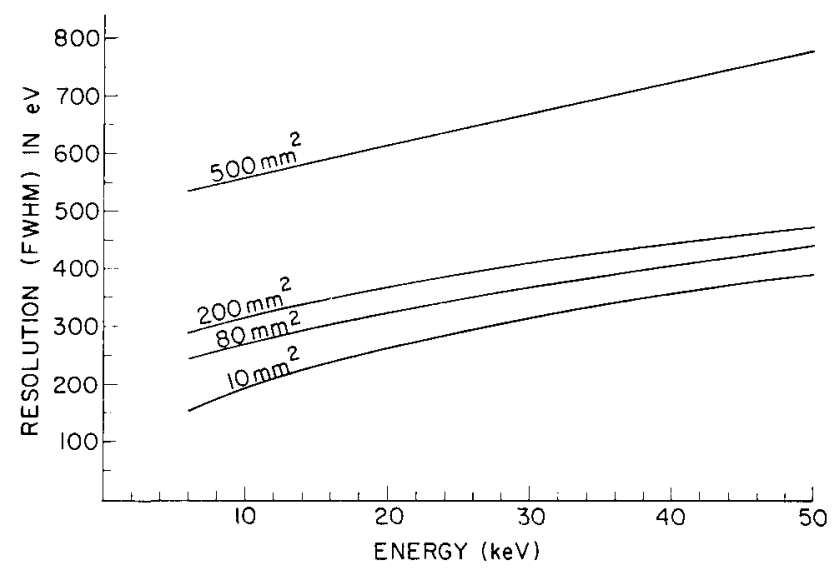

Fig. 18. The resolutions of silicon solid state detectors with various areas (Frankel and Aitken, 1970).

continuum; these cannot be observed efficiently with the higher resolution crystal spectrometers. The solid state detector and objective grating spectrometer are complementary; the solid state detector has superior resolution and efficiency at higher X-ray energies, whereas the objective grating spectrometer has superior resolution and efficiency at lower energies.

\section{Future Developments}

We have reviewed most of the instruments which have been used or proposed for $\mathrm{X}$-ray astronomy, and it is appropriate to consider the prospects for spectroscopy in this field. At this time there are no positive results indicating sharp spectral features from X-ray sources, although only Sco-X1 has been studied with high resolution. A number of low resolution survey instruments will be launched during the next few years, including instruments on OSO-H, OSO-1, SAS-C, UK-5, and HEAO-A and HEAO-B. The NRL proportional counter experiment on HEAO-A and the Columbia 
AS \& E Cal. Tech. focussing collector on the same spacecraft will be the most sensitive of these instruments. These experiments will extend our present observations to a larger energy range and to sources a thousand times weaker than present observational limits. This will be particularly important for new types of sources which may be discovered, and for extra galactic objects similar to established sources but too faint to be observed at this time. There will be also a number of relatively small high resolution crystal instruments flown during this period, including experiments on the OSO 1; ANS and UK-5 satellites, and these probably will detect interesting spectral features in the stronger sources. The Angel-Walker experiment on HEAO-B will be the first high resolution instrument with sufficient sensitivity to perform meaningful studies of a large number of sources.

The first experiment package having the general characteristics and flexibility of a ground based visible light observatory will be flown on the HEAO-C spacecraft now scheduled for launch in 1977. The experiments for this observatory are only partially selected at this time, but will include at least two focussing X-ray telescopes, and various interchangeable focal plane instruments. The use of two large area telescopes will permit simultaneous measurements of related phenomena, such as the temporal behaviour of a source line and continuum. One telescope will have a resolution of an arc second and about $1000 \mathrm{~cm}^{2}$ collecting area, and one will have about $2000 \mathrm{~cm}^{2}$ collecting area but about $30^{\prime \prime}$ resolution. The focal plane instruments will include sensitive imaging devices with arc second resolution, a solid state detector, and an objective grating spectrometer for low resolution measurements of the fainter objects or sources with broadened spectral features. The instruments also will include high resolution crystal spectrometers sufficiently sensitive to study any of the presently known sources. The HEAO-C experiment will be able to achieve arc second locations and low or moderate resolution spectral measurements of any sources having sufficient intensities to be found prior to its flight by other presently planned experiments. These measurements also will be possible for sources which have the same intrinsic luminosity as the presently known galactic sources but located anywhere in the local group of galaxies; in many cases such sources would be too closely spaced to be resolved by the lower angular resolution instruments which will fly earlier. This will permit absolute luminosity measurements free of source distance uncertainties, will allow the correlation between X-ray source types and galactic structure to be studied, and possibly will enable meaningful evolutionary models to be constructed. We believe that these telescopes will be as important to X-ray astronomy as the first large visible light or radio telescopes were in these areas; we believe that it will cause a qualitative change in the character of the science.

\section{References}

Alkhazov, G. D., Komar, A. P., and Vorab'ev, .: 1967, Nucl. Instr. Meth. 48, 1.

Angel, J. R. P.: 1969, Nature 226, 734.

Angel, J. R. P. and Weisskopf, M. C.: 1970, Astron. J. 75, 231.

Blake, R. L.: 1970, Adv. X-ray Analysis 13, 352. 
Bunner, A. N., Coleman, P. L., Kraushaar, W. L., McCammon, D., Palmieri, T. M., Shilepsky, A. and Ulmer, M.: 1969, Nature 223, 1222.

Byrne, J.: 1962, Proc. Roy. Soc. Edinburgh A66, 33.

Charles, M. W. and Cooke, B. A.: 1968, Nucl. Instr. Meth. 61, 31.

Cottrell, T. L. and Walker, I. C.: 1961, Faraday Soc. Trans. 61, 1591.

Cornell, C. M., Grader, R. J., Harri, J. G., Hill, R. W., Rumble, R. P., and Salmi, D. S.: 1968, Rev. Sci. Instr. 39, 951.

Frankel, R. S. and Aitken, D. W.: 1970, Appl. Spectr. 24, 557.

Frost, K. J., Rothe, E. D., and Peterson, L. E.: 1966, J. Geophys. Res. 71, 4079.

Gabriel, A. H. and Jordan, C.: 1969, Nature 221, 947.

Giaconni, R. and Rossi, B.: 1960, J. Geophys. Res. 65, 773.

Giaconni, R., Reidy, W. P., Vaiana, G. S., Van Speybroeck, L. P., and Zehnpfennig, T. F.: 1969, Space Sci. Rev. 9, 3.

Gorenstein, P. and Mickicwicz, S. : 1968, Rev. Sci. Instr. 39, 816.

Gott, R., Parkes, W., and Pounds, K. A.: 1970, Nucl. Instr. Meth. 81, 152.

Gursky, H. and Zehnpfennig, T.: 1966, Appl. Opt. 5, 875.

Harris, T. J. and Mathieson, E.: 1971, Nucl. Instr. Meth. to be published.

Jaklevis, J. M. and Goulding, F. S.: 1971, I.E.E.E. Trans. Nucl. Sci. NS-18, 187.

Kesterbaum, H., Angel, J. R. P., and Novick, R.: 1971, Astrophys. J. 164, 187.

Liefeld, R. J., Hanzaly, S., Kirby, T. B., and Mott, D.: 1970, Adv. X-Ray Anal. 13, 373.

Mathieson, E. and Sanford, P. W.: 1964, Proc. Intern. Symp. on Nucl. Electronics, Paris, (1963) (ENEA, 1964), p. 65.

Mathieson, E. and Harris, T. J.: 1970, Nucl. Instr. Meth. 88, 181.

Novick, R.: 1971 , private communication.

Parkes, W., Gott, R., and Pounds, K. A.: 1970a, I.E.E.E. Trans. Nucl. Sci. NS-17, 360.

Parkes, W., Gott, R., and Pounds, K. A.: 1970b, I.E.E.E. Trans. Nucl. Sci. NS-17, 367.

Peterson, L. E., Pelling, R. M., and Matteson, J. L.: 1971, Contribution to the 14th COSPAR Plenary Meeting, Seattle, Washington, June.

Pounds, K. A.: 1971, Nature (Phys. Sci.) 229, 175.

Ricke, G. R. and Gomes, J. J.: 1969, Rev. Sci. Instr. 40, 227.

Ruderman, I. W. and Michelman, B.: 1968, Isomet. Corp. Report, Present Status of X-Ray Analyzer Crystals.

Schnopper, H. W. and Kalata, K.: 1969, Appl. Phys. Letters 15, 134.

Smith, D. G. and Pounds, K. A.: 1968, I. E. E. E. Trans. Nucl. Sci. NS-15, 541.

Speer, R. J., Peacock, N. J., Waller, and Osborne, P. J.: 1970, J. Phys. E (Sci. Instr.) (GB) 3, 143.

Tananbaum, H. and Kellogg, E. M.: 1970, I.E.E.E. Trans. Nucl. Sci. NS-17, 97.

Tucker, W.: 1967, Astrophys. J. 148. 754.

Tucker, W. and Koren, M.: 1971, Astrophys. J. 168, 283.

Van Speybroeck, L. and Chase, R.: 1971, J. Appl. Opt.

Vierling, J., Gilfrich, J. V. Birks, L. S. : 1969, Appl. Spectrosc. (U.S.A) 23, 342.

Walker, A. B. C. and Rugge, H. R.: 1971, private communication.

Wolter, H.: 1952a, Ann. Physik 10, 94.

Wolter, H.: 1952b, Ann. Physik 10, 286.

Zulliger, H. R. and Aitken, D. W.: 1970, I.E.E.E. Trans, Nucl. Sci. NS-17, 187.

Zulliger, H. R., Middleman, L. M., and Aitken, D. W.: 1969, I.E.E.E. Trans. Nucl. Sci. NS-16, 47. 